\title{
Extensão cranial da veia safena parva: quando o fluxo caudal é normal
}

\author{
Cranial extension of the small saphenous vein: when caudal flow is \\ normal
}

\section{André Paciello Romualdo, Roberto de Moraes Bastos, Mathias Fatio, Alessandro Cappucci, Solange Augusta Munhoz Mariana, Érika Narahashi, Alberto Lobo Machado, Eduardo Hideki Tokura*}

\begin{abstract}
Resumo
A extensão cranial da veia safena parva se destaca pelas inúmeras variações anatômicas e diferentes padrões de fluxo que podem ser observados, descritos em trabalhos envolvendo dissecções pós-morte ou cirúrgicas, flebografias e Doppler, que denotam a formação embriológica mais precoce e complexa em relação à safena magna. A observação de um tipo específico de extensão cranial da safena parva onde o sentido das valvas é contrário ao habitualmente observado foi primeiramente caracterizada por Carlo Giacomini, sendo o fluxo caudal nesses casos de aspecto normal sem sinal de incompetência valvar. Este artigo demonstra os padrões anatômicos e de fluxo que podem ser caracterizados na veia safena parva, contribuindo para que aspectos normais do seu fluxo não sejam confundidos com incompetência valvar.
\end{abstract}

Palavras-chave: Veia, Doppler, refluxo.

\section{Introdução}

O estudo por ultrassonografia com Doppler representa importante ferramenta diagnóstica de patologias vasculares, particularmente em relação às veias dos membros inferiores. Dentre os diversos segmentos venosos, a extensão cranial da veia safena parva se destaca pelas inúmeras variações anatômicas e diferentes padrões de fluxo que podem ser observados, sendo o correto conhecimento de sua anatomia e fisiologia fundamental para um diagnóstico adequado.

O objetivo deste estudo é salientar que, dentre as possíveis variações anatômicas descritas, uma em particular apresenta fluxo com sentido caudal, aspecto que pode ser bem caracterizado ao estudo de ultrassonografia com Doppler.

\begin{abstract}
Cranial extension of the small saphenous vein is of special interest due to the number of anatomical variations and different blood flow patterns observed, which have been described in investigations including postmortem or surgical dissections, phlebographies, and Doppler studies, indicating an earlier and more complex embryological development in relation to the great saphenous vein. A specific type of cranial extension of the small saphenous vein, where a reverse flow is often observed in the valves, was first characterized by Carlo Giacomini. In these veins, caudal flow is normal, with no evidence of valvular incompetence. This article describes anatomical and blood flow patterns found in the small saphenous vein, thus contributing to avoid that normal blood flow aspects are misinterpreted as valvular incompetence.
\end{abstract}

Keywords: Vein, Doppler, reflux.

\section{Histórico}

O primeiro anatomista a descrever uma extensão cranial da veia safena parva foi Josef Hyrtl em 1864, quando introduziu o termo "veia fêmoro-poplítea" para uma veia superficial da face posterior da coxa, termo esse incluído em 1936 em um dos consensos de terminologia anatômica da época, a Jenaer Nomina Anatomica, sendo depois substituído na nomenclatura oficial nas edições da Nomina Anatomica por "veia subcutânea femoral posterior" e mais tarde por "veia marginal medial"1,2.

Dentre os anatomistas do século XIX, Carlo Giacomini destacou-se com seu trabalho realizado em $1873^{3}$, quando descreveu novos tipos de terminação da safena parva, sua relação com as fáscias da coxa, demonstrando que se tratava da própria safena estendendo-se no segmen-

*Fleury Medicina e Saúde, São Paulo, SP.

Não foram declarados conflitos de interesse associados à publicação deste artigo. Artigo submetido em 24.06.08, aceito em 21.01.09.

J Vasc Bras. 2009;8(2):166-170.

Copyright (C 2009 by Sociedade Brasileira de Angiologia e de Cirurgia Vascular 
to supragenicular e não veias superficiais anômalas como anteriormente sugerido, concluindo que "o ramo ascendente que sai da veia safena parva, ao invés de ser considerada uma anomalia, por causa da sua frequência extrema, deveria ser considerada um estado normal"3. Outra contribuição original foi a descoberta de dois arranjos valvares opostos: o primeiro permitindo apenas fluxo cranial quando da existência de uma anastomose intersafena e o segundo permitindo fluxo caudal em direção à junção safenopoplítea, funcionando como uma tributária do segmento proximal da coxa ${ }^{1}$.

O termo em vigência atualmente, extensão cranial da veia safena parva, surgiu de um consenso entre radiologistas, cirurgiões vasculares e anatomistas, reunidos no XIV Congresso da União Internacional de Flebologia, realizado em Roma em 2001 e avalizado por órgãos oficiais de anatomia, a Federação Internacional de Associação de Anatomistas e o Comitê Federativo em Terminologia Anatômi$\mathrm{ca}^{4,5}$. De acordo com tal consenso, o termo extensão cranial da safena parva inclui qualquer terminação da safena parva acima da prega poplítea, incluída a veia de Giacomini.

\section{Anatomia}

A veia safena parva cursa no compartimento safeno, definido pelas fáscias muscular, mais profunda, e safena, mais superficial (Figura 1). Na margem inferior da fossa poplítea, o compartimento safeno deixa de existir porque a fáscia muscular adere às fáscias dos músculos gastrocnêmios para inserir-se nos côndilos femorais. A fáscia poplí-

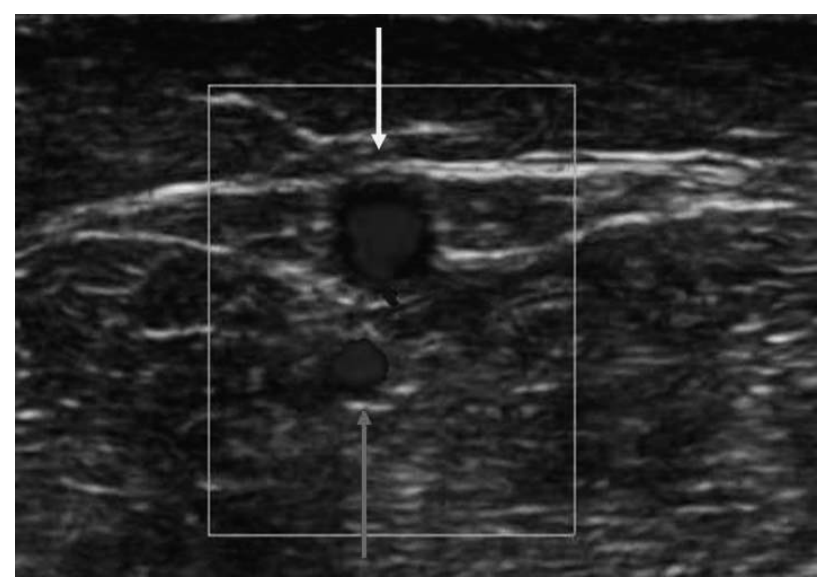

Figura 1 - Veia safena parva infragenicular (seta branca) no interior do compartimento safeno; profundamente ao compartimento safeno corre a veia intergemelar (seta cinza) tea representa a extensão da fáscia safena, reforçada por fibras das fáscias dos músculos gastrocnêmios, semitendinoso e bíceps femoral ${ }^{6}$.

Quando se estende acima da prega poplítea, a veia safena parva aprofunda-se juntamente com a fáscia safena, passando a correr entre os ventres dos músculos bíceps femoral e semitendinoso em um trajeto subfascial (Figura 2).

Os tipos de terminação da safena parva têm sido objeto de estudo por mais de um século, a princípio por dissecções em cádaver ${ }^{3,7}$, mais recentemente por exames ultrassonográficos com Doppler ${ }^{8-12}$.

De forma geral esses estudos confirmam os aspectos anatômicos propostos por anatomistas como Charles Kosinski ${ }^{7}$, que em 1926 referendou o trabalho de Giacomini em um estudo de dissecção de 124 pernas dividindo de maneira bastante didática as terminações da safena parva em diferentes padrões, como mostram as Figuras 3, 4 e 5.

\section{Fisiologia}

Enquanto os aspectos anatômicos da veia safena parva já foram amplamente discutidos, pouca ou nenhuma ênfase tem sido dada à possibilidade de fluxo invertido em um padrão específico de extensão cranial da safena parva. Isso ocorre nos casos em que existe junção safeno-poplítea e que é observada veia de pequeno calibre de trajeto subfascial que se inicia em pequenas veias subcutâneas no terço superior/médio da coxa posterior (padrão 1A). Tal extensão cranial apresenta fluxo de sentido caudal, já que essa veia se comporta como tributária da face posterior da coxa (Figuras 6 e 7). Nas palavras do próprio

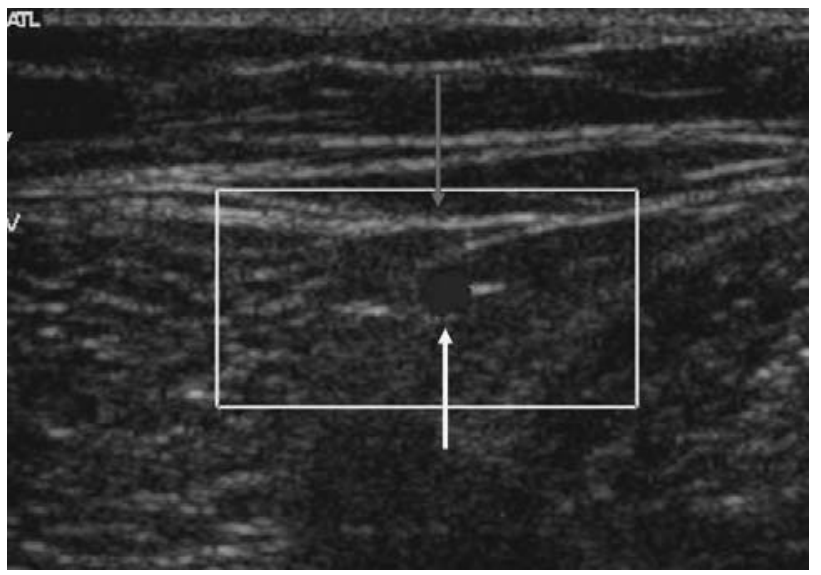

Figura 2 - Veia safena parva supragenicular (seta branca) corre subjacente à fáscia safena (seta cinza) 


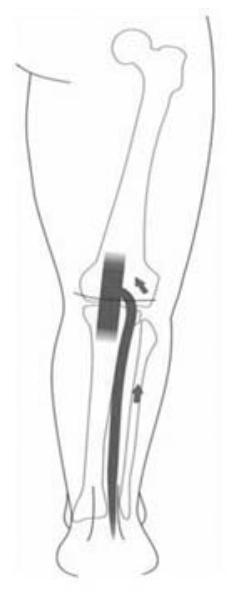

Padrão 1A

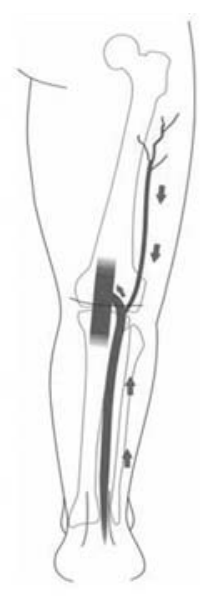

Padrão $1 \mathrm{~A}$

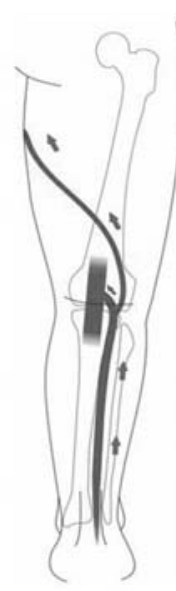

Padrão 1B
Figura 3 - Terminações da safena parva: padrão 1A) terminação exclusiva na veia poplítea, a crossa pode receber uma pequena veia superficial posterior que se inicia no terço superior ou médio da coxa, que eventualmente apresenta comunicações com veias musculares profundas da coxa; padrão $1 \mathrm{~B}$ ) terminação divide-se em dois ramos, um drena na veia poplítea e o outro drena na veia safena magna

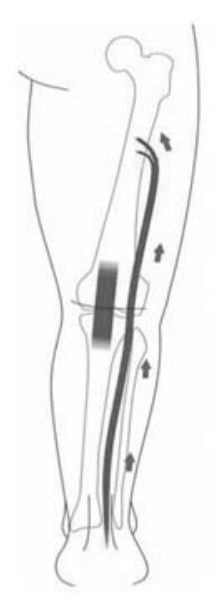

Padrão 2A

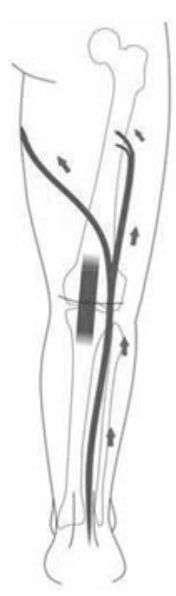

Padrão 2B
Padrão 2C

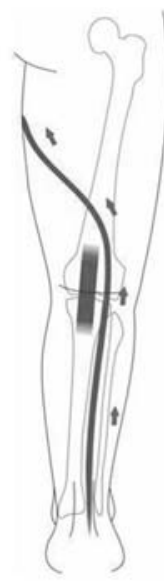

Figura 4 - Terminações da safena parva: padrão 2A) terminação em veias posteriores profundas da coxa; padrão $2 \mathrm{~B}$ ) terminação divide-se em dois ramos, um drena em veia profunda posterior e outro drena na veia safena magna; padrão 2C) terminação direta na veia safena magna

Giacomini, “a veia safena parva apresenta junção safeno-poplítea [...] esse ramo termina no tecido subcutâneo/subfascial na porção posterior e superior da coxa. [...] obviamente leva o sangue para a safena parva, já que existia um par de valvas arranjadas de modo a prevenir o

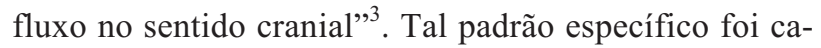
racterizado em 14\% dos membros estudados por ele e $23 \%$ dos membros estudados com Doppler por Oliveira et

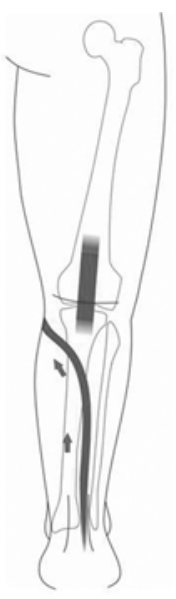

Padrão $3 \mathrm{~A}$

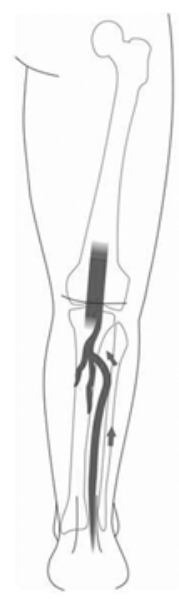

Padrão 3B
Figura 5 - Terminações da safena parva: padrão 3A) terminação na veia safena magna abaixo da prega poplítea; padrão $3 \mathrm{~B}$ ) terminação nas veias gastrocnêmias

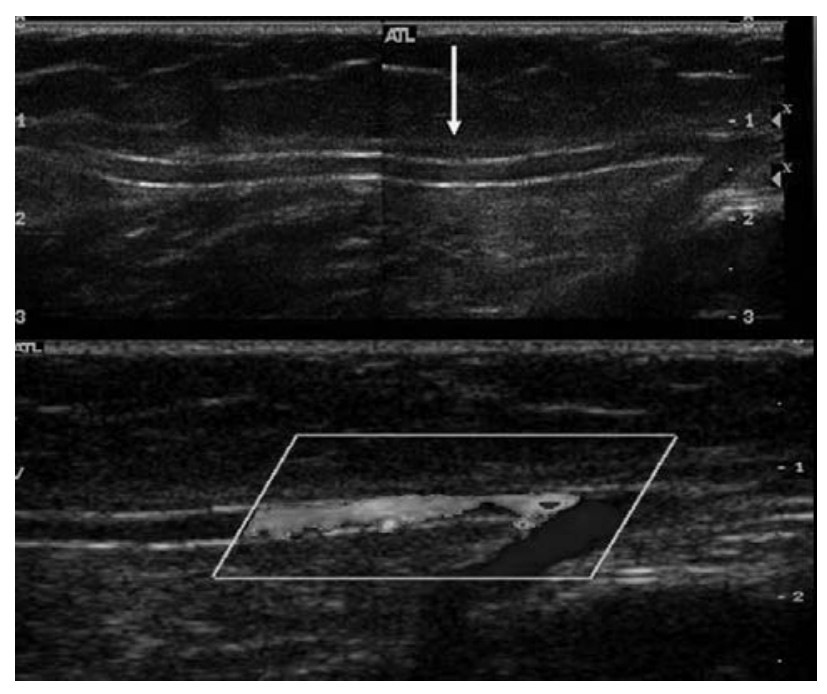

Figura 6 - Extensão cranial da veia safena parva, padrão $1 \mathrm{~A}$ (seta); o fluxo em cinza claro ao estudo Doppler indica o sentido caudal, drenando na crossa da veia safena parva com fluxo em cinza escuro indicando sentido cranial

al. ${ }^{8}$. Nos demais estudos anatômicos não se fez menção à sua frequência, cuja presença foi ou omitida ou incorporada ao padrão em que ocorre terminação exclusiva na veia poplítea.

Nos segmentos intersafenas, as valvas estão arranjadas de modo a levar o sangue da safena parva até a safena magna (padrões 1B, 2B, 2C e 3A). A veia de Giacomini, que é formada por um segmento subfascial e outro subcutâneo, apresenta fluxo no sentido cranial (Figura 8). Conforme o próprio Giacomini, "podem ser encontradas valvas na sua porção subfascial e constantemente 
2 ou 3 pares na porção subcutânea próximo à junção com a safena magna. [...] essas valvas estão arranjadas de modo a prevenir o refluxo da safena magna para a safena parva"”.
As demais extensões craniais citadas por Kosinski ${ }^{7}$, que terminam em veias profundas da coxa (padrão $2 \mathrm{~A}$ ) por meio de perfurantes ou aprofundando-se em meio à musculatura, apresentam sempre fluxo de sentido cranial (Figura 9).

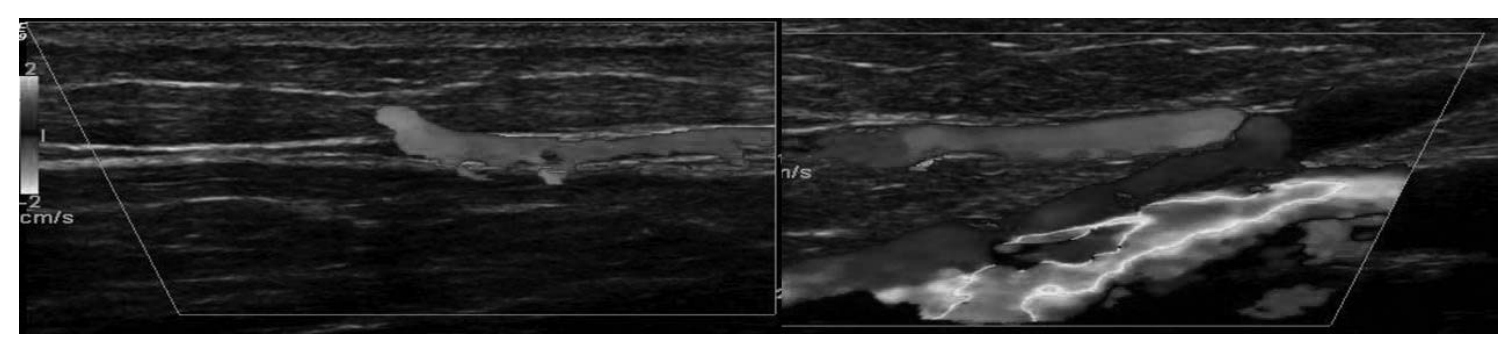

Figura 7 - Outro exemplo de extensão cranial da veia safena parva, padrão 1A, com fluxo caudal ao estudo Doppler

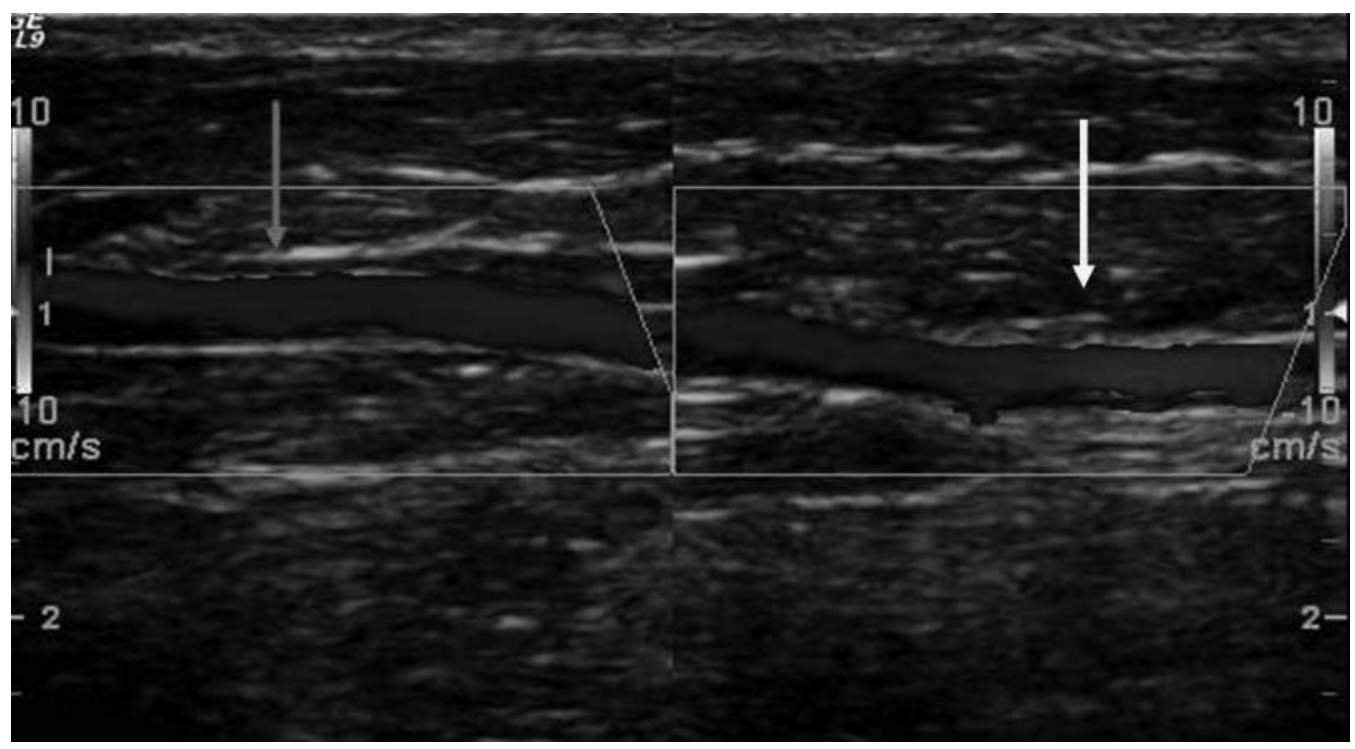

Figura 8 - Veia de Giacomini com segmentos subfascial (seta branca) e subcutâneo (seta cinza) com fluxo ao estudo Doppler indicando sentido cranial (área mais escura dentro dos quadrados)

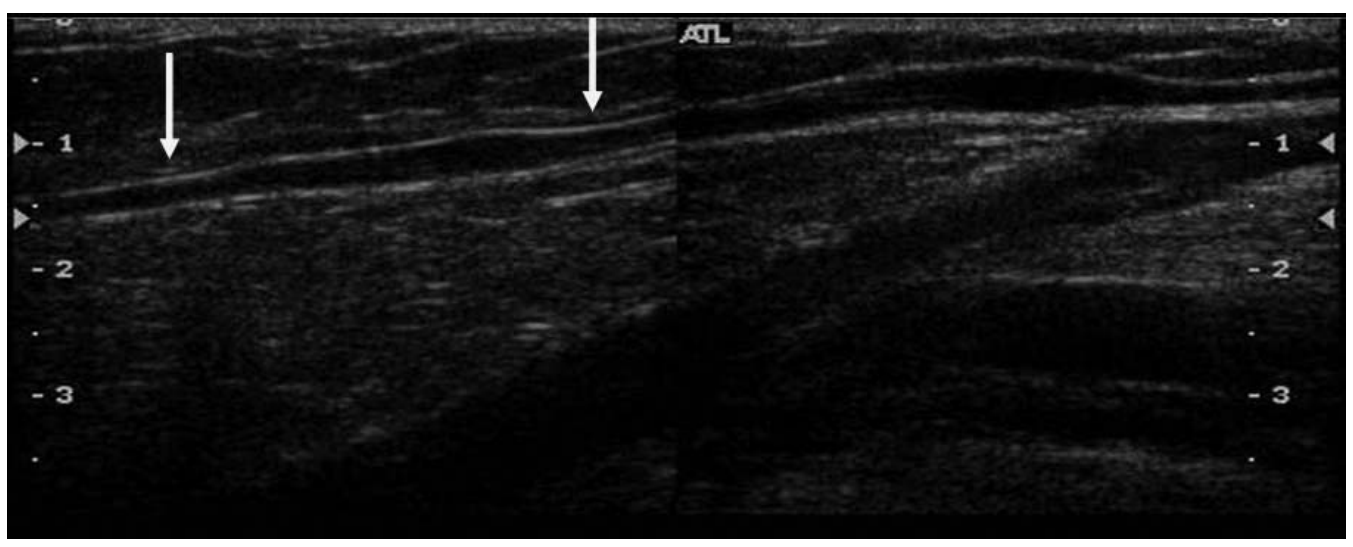

Figura 9 - Extensão cranial da safena parva, padrão 2A; crossa da safena parva não caracterizada e extensão cranial aprofunda-se em meio à musculatura da coxa (setas) 


\section{Conclusão}

Desde o século XIX, inúmeros estudos anatômicos têm descrito os variados tipos de terminação anatômica da veia safena parva, destacando-se o trabalho de Giacomini, que caracterizou o padrão valvar em tais segmentos venosos. A observação de um tipo específico de extensão cranial da safena parva onde o sentido das valvas é contrário ao habitualmente observado deve ser de conhecimento do examinador, pois a caracterização de fluxo caudal nesses casos representa achado normal e não uma incompetência valvar, como corriqueiramente e de forma equivocada é interpretado.

\section{Referências}

1. Georgiev M, Meyers K, Belcaro G. The tigh extension of the lesser sapfenous vein: From Giacomini's observations to ultrasound scan imaging. J Vasc Surg. 2003;37:558-63.

2. Georgiev M. The femoropopliteal vein: ultrasound anatomy, diagnosis, and office surgery. Dermatol Surg. 1996;22:5762.

3. Giacomini C. Osservazioni anatomiche per servire allo studio della circolazione venosa delle estremita inferiore. Gior R Accad Med Torino. 1873;13:109-215.

4. Caggiati A, Bergan J, Gloviczki P, et al. Nomenclature of the veins of the lower limbs: an international interdisciplinary consensus statement. J Vasc Surg. 2002;36:416-22.
5. Caggiati A, Bergan J, Gloviczki P, et al. Nomenclature of the veins of the lower limb: extensions, refinements, and clinical application. J Vasc Surg. 2005;41:719-24.

6. Caggiati A. Fascial relationships of the short saphenous vein. J Vasc Surg. 2001;34:241-6.

7. Kosinski C. Observations of the superficial venous system of the lower extremity. J Anat. 1926;60:131-42.

8. Oliveira A, Vidal EA, França GJ, Toregiani J, Timi JR, Moreira RC. Estudo das variações anatômicas da terminação da veia safena parva pelo eco-Doppler colorido. J Vasc Bras. 2004;3(3):220-30.

9. Delis KT, Knaggs AL, Khodabakhsh P. Prevalence, anatomic patterns, valvular competence, and clinical significance of the Giacomini vein. J Vasc Surg. 2004;40:1174-83.

10. Engel AF, Davies G, Keeman JN. Preoperative localization of the saphenofemoral junction with duplex scanning. Eur J Vasc Surg. 1991;5:507-9.

11. Somjen GM, Royle JP, Fell G, Roberts AK, Hoare MC, Tong Y. Venous reflux patterns in the popliteal fossa. J Cardiovasc Surg. 1992;33:85-91.

12. Engel AF, Davies G, Keeman JN, von Dorp TA. Colour flow imaging of the normal short saphenous vein. Eur J Vasc Surg. 1994;8:179-81.

Correspondência:

André Paciello Romualdo

Rua Martiniano de Carvalho, 836/32, bloco 1, Bairro Bela

Vista

CEP 01321-000 - São Paulo, SP

Tel.: (11) 3283.0980

E-mail: andrepaciello@hotmail.com 